\title{
Prediction of Enterprise Economic Activity Behavior Based on Neural Network and ARIMA Hybrid Model
}

\author{
Zeyu $\operatorname{Lin}^{1}$ and Shuai $\mathrm{Li} \mathbb{D}^{2}$ \\ ${ }^{1}$ Hong Kong Baptist University, Kowloon Tong, Hong Kong 999077, China \\ ${ }^{2}$ Central University of Finance and Economics, Beijing 100081, China \\ Correspondence should be addressed to Shuai Li; cufejwc_ls@cufe.edu.cn
}

Received 4 September 2021; Revised 21 October 2021; Accepted 25 October 2021; Published 15 November 2021

Academic Editor: Han Wang

Copyright (c) 2021 Zeyu Lin and Shuai Li. This is an open access article distributed under the Creative Commons Attribution License, which permits unrestricted use, distribution, and reproduction in any medium, provided the original work is properly cited.

\begin{abstract}
Enterprise economy refers to the comprehensive situation reflected in the gross product, production scale, total production and efficiency, technological content, marketing means, and so on; under certain social conditions, enterprises use resources obtained by law to engage in economic activities. Under the guidance of consciousness or culture, enterprises use "legally obtained resources" to promote economic development. Enterprise economy is affected by manpower, capital, management, operation, policy, and other aspects. In the context of the rapid development of big data in the current era, this paper proposes a prediction model of enterprise economic activity behavior based on neural network and ARIMA by investigating a variety of artificial intelligence models and verifies its feasibility. Commodity circulation enterprises have a more urgent demand for the development of business audit due to their operation characteristics. Therefore, this paper takes commodity circulation enterprises as representatives and predicts business audit in the big data environment based on the model proposed in this paper.
\end{abstract}

\section{Introduction}

Prediction is a study of what has not happened and what is not clear in advance, speculating and realistically studying the results of things that should happen. Since Lapebest and Farbe first applied artificial neural network technology to forecast, neural network method has been paid more and more attention. Many experts and scholars at home and abroad have done a lot of research on the application of neural network in prediction and achieved certain results [1].

In the economics field, as an important branch of forecasting field, economic forecasting has attracted much attention. Although traditional enterprise economic forecasting methods have advantages, with the complexity of economic management problems, these traditional methods cannot meet the requirements of scientific management and correct management. Neural network technology provides a new research conception and method for forecasting research. Since the previous research has achieved certain research results, it has become a valuable research topic to apply neural network to the research of enterprise economic forecasting, which forms the basis and idea of this topic selection. However, because the economic operation system is a complex system, the success of neural network technology in economic prediction and alarm is obviously a huge systematic project [2]. Therefore, the research in this field is mostly based on exploratory nature. With the deepening of financial sharing and industrial property integration, the management and supervision of commodity circulation enterprises has become a trend. At this time, enterprises can develop an embedded audit system that conforms to their own business characteristics and realize real-time monitoring and alarm such as the occurrence, collection, and analysis of business data, so as to truly monitor before and after the event at the same time [3].

Aiming at the lack of previous studies, this paper puts forward some views and ideas of its own and establishes a mixed model of enterprise economic activity action prediction based on neural network and ARIMA. On this basis, an empirical study is carried out by using actual data. It lays a good foundation for further research in the future. 
CPU, hard disk utilization, and resource consumption often have a great impact on the response speed of programs, and insufficient memory may even lead to program crash. Performance models pay more attention to these contents. We propose a method based on memory measurement and derived measurement to narrow the gap and predict the impact of resources. In the past, there were many models to solve this problem. However, the accuracy is too low, so we use the performance model generator to automatically extract memory behavior and evaluate it with industry benchmarks. The results are satisfactory. It can play a role in capacity planning (internal), cost forecasting (cloud), and so forth and can also be used for continuous model-based evaluation of memory usage of enterprise architecture [4]. We study the potential factors that affect the economic cycle and turbulence and take the venture capital market as a case to study the timing in organizational behavior. It is shown that once an enterprise carries out more frequent business activities or transactions in a short time, sudden effects will occur, which is the potential factor. Similarly, when the memory effect of organization occurs, it can predict the future behavior. From this, we find the changing characteristics of organizational behavior and economic system, which makes researchers think it is necessary to extend the study of human dynamics to the study of organizational dynamics [5].

Performance appraisal is very important for enterprises. With the expansion of enterprise scale and the increase of employees, how to match the key characteristics of employees' performance and how to accurately identify potential employees are the key issues of research. The application of deep learning to analyze data sets and identify and predict employees' potential and reasons for turnover is significantly improved compared with traditional machine learning methods [6]. We build an employee topology diagram based on interactive network and then use a large amount of collected employee data to communicate and analyze with employee data from all walks of life to get its key features. Finally, compared with the traditional model, the accuracy and AUC indicators are improved. Because of its characteristics, the design of composite cap stiffening plate will involve a large number of geometric and material parameters. Therefore, we put forward an analysis tool with high analysis and calculation efficiency based on artificial neural network for high-efficiency calculation and analysis. The tool uses the training set, verification set, and test set of neural network and compresses the original characteristics and then establishes back-propagation neural network to predict buckling and finally the test is performed. Experiments show that the neural network can effectively predict the buckling and ultimate load of composite cap-ribbed plates under in-plane shear action, which further reflects that the analysis is of great significance for practical design [7]. We use 1000 small businesses as a sample to study the potential of small business default prediction. Combining industry priority payment behavior with Kohonen map, two prediction models based on financial ratio and payment behavior related variables are calculated. The results show that the prediction accuracy of Kohonen map is inversely proportional to the enterprise scale; if small businesses add variables related to payment, the accuracy can be greatly improved [8]. The previously mentioned research methods are also good, but when many enterprises predict their economic activities, they do not study the fusion of various algorithms, and there are defects in the prediction accuracy.

\section{Hybrid Model of Neural Network and ARIMA}

2.1. Neural Network Model. Artificial neural network is referred to as neural network. As a branch of artificial intelligence, it has been widely used in model recognition, optimization, intelligent robot, control, prediction, detection, and so on. At present, there are many ways to build neural networks, including neural network models based on mathematical methods, neural network models based on thermodynamic methods, and neural network models based on fuzzy and chaotic methods. It has become an important direction in the field of science. The basic characteristics of neural network make it have unique advantages. When predicting the data with few training samples, it can get the network threshold and network weight quickly and accurately and has strong learning and fitting ability. Neuron is the most basic unit of neural network. It is the simplest neural network, which can process information nonlinearly. It can have multiple inputs but only one output. Its structure is shown in Figure 1, which consists of three parts: input, activation function, and output [9]. $x_{1}, x_{2}, \ldots, x_{r}$ are $r$ input parameters, $\omega_{i}(i=1,2, \ldots, r)$ and $b$ are the weights and biases of neural network connection, respectively, $n=\sum \omega_{i} x_{i}+b$ will be brought into an activation function $f(\cdot)$ as input, and the final output is $a=f\left(\sum \omega_{i} x_{i}+b\right)$.

In the input neuron structure proposed in Figure 1, economic indicators are used as input variables to learn and train neurons. Generally, the input economic index data needs to be preprocessed, and the relevant processing process has been given in the article.

Then, a neural network will be obtained by connecting a certain number of neurons according to the required structure, in which the activation function is the key to realize the nonlinear mapping of the neural network. The commonly used activation functions are as follows:

(1) Sigmoid function:

$$
f(x)=\frac{1}{1+e^{-x}} .
$$

(2) Tanh function:

$$
f(x)=\frac{e^{x}-e^{-x}}{e^{x}+e^{-x}}
$$

(3) Threshold function:

$$
\begin{array}{r}
\text { (a) } f(x)= \begin{cases}1, & x \geq 0, \\
0, & x<0,\end{cases} \\
\text { (b) } \operatorname{sgn}(x)= \begin{cases}1, & x \geq 0, \\
-1 & x<0 .\end{cases}
\end{array}
$$




$$
f(x)=\max (0, x) .
$$

2.2. BP Neural Network. Feedforward neural network, referred to as BP neural network for short, was established in 1986. When the training error is obtained, the network propagates it backwards, and the parameters in the network are modified in this way. When the sum of squares of the error meets the requirements of network accuracy, the iteration will stop. Its signal propagation is unidirectional; that is, the signal of each layer only affects the next layer but has no influence on the upper layer and other layers [10]. It transforms the input and output of experimental samples into a nonlinear optimization problem, and the gradient descent algorithm is adopted. In fact, for this network, it is worth noting that it can effectively approximate any nonlinear function and only needs one hidden layer. It can be seen that this network has strong learning ability and strong feasibility, so it has become the most widely used artificial neural network. Its topology is shown in Figure 2.

The modeling steps of BP neural network are as follows:

Step 1. Data preprocessing:When forecasting, if the values of the data in the samples differ a lot, it is easy to have numerical problems. In order to solve these problems, it is necessary to normalize the original data. The normal way of normalization is

$$
Y_{i}=\frac{x_{i}-x_{\min }}{x_{\max }-x_{\min }}, \quad i=1,2, \ldots, n
$$

After the original data is normalized, the data $Y_{i}, x_{i}$ are the input values, and $x_{\max }, x_{\min }$ are the maximum and minimum values of the normalized input sequence, respectively. It is worth noting that, in order to obtain the final predicted value of the model, the predicted value of the network needs to be denormalized in the subsequent process:

$$
x_{i}=Y_{i} *\left(x_{\max }-x_{\min }\right)+x_{\min } .
$$

Step 2. Determining the structure of neural network: According to the characteristics of data, the optimal number of structural layers and the number of nodes of each structural layer needed by the built neural network model are determined [11]. For the problem of determining the number of nodes in each layer, it is important and difficult to determine the number of nodes $N$ needed in the hidden layer. At present, there is no accurate method for this problem, which cannot be generalized. People need to try many times to finally determine the value of $N$ which makes the prediction effect of the model optimal. It should be noted that the value of $N$ should not be too large or too small. If the number of nodes is too large, the training process will be too complicated and the training time will be increased. If the number of hidden layer nodes is too small, the model cannot learn enough nonlinear features. The value of $N$ can be preliminarily determined by the following empirical formula, and people need to try to compare many times near the determined number of nodes and finally determine the value of the number of nodes $n$.

$$
\left\{\begin{array}{l}
n=\sqrt{N M} \\
n=\log _{2} N \\
n=\sqrt{N+M}+a, \quad a \in[1,10]
\end{array}\right.
$$

where $n$ represents the number of nodes in network training; formula (7) uses three formulas to describe the value process of $n$, which is difficult to determine in the training process. Different ways lead to different results and have different influences on the performance of the algorithm. In order to affect the overall algorithm effect, three formulas are used to describe $n$, and the formula value of $n$ is determined according to the sample size and the corresponding algorithm requirements.

Step 3. Determining the activation function: The hidden layer and the output layer need to determine the activation function, and there are many choices for the activation function $f(x)$, such as tansig, logsig, purelin, and many other functions.

Step 4. Initializing weights, thresholds, and learning rates

Step 5. Forward-propagation calculation: The normalized data is input and propagated forward through the determined network to obtain the following:

Hidden layer output $H_{j}$ :

$$
H_{j}=f\left(\sum_{i=1}^{n} \omega_{i j}+a_{j}\right), \quad j=1,2, \ldots, L,
$$

$\omega_{i j}$ is the weight from the input layer to the hidden layer, $a_{j}$ is the hidden layer threshold, and $L$ is the number of hidden layer nodes.

Output layer output $O_{k}$ :

$$
O_{k}=\sum_{i=1}^{n} \omega_{j k} H_{j}+b_{k}, \quad k=1,2, \ldots, m,
$$

$\omega_{j k}$ is the weight from hidden layer to output layer and $b_{k}$ is the threshold of output layer.

Step 6. Error calculation

$$
e_{k}=Y_{k}-O_{k}, \quad k=1,2, \ldots, m
$$

Step 7. Weight updating: According to the prediction error $e_{k}$ calculated by the above formula, the weights $\omega_{i j}$ and $\omega_{j k}$ in the established neural network are updated and adjusted.

$$
\begin{aligned}
& \omega_{i j}=\omega_{i j}+\eta H_{j}\left(\left(1-H_{j}\right) x_{i} \sum_{k=1}^{m} \omega_{i k} e_{k}\right), \\
& \omega_{j k}=\omega_{j k}+\eta H_{j} e_{k} .
\end{aligned}
$$


Step 8. Threshold updating: The thresholds $a_{j}$ and $b_{k}$ are updated according to the prediction error $e_{k}$.

$$
\begin{aligned}
& a_{j}=a_{j}+\eta H_{j}\left(1-H_{j}\right) \sum_{k=1}^{m} \omega_{j k} e_{k}, \\
& b_{k}=b_{k}+\eta e_{k} .
\end{aligned}
$$

Step 9. Judging whether the iteration can be terminated after obtaining new weights and biases; if not, return to step 5 to iterate and update parameters repeatedly until the minimum mean square error is less than the set value, and the BP neural network modeling is finished.

\subsection{ARIMA Model}

\subsubsection{Time Series}

(1) Definition of Time Series. Time series refers to a series of numbers arranged by the values of the same statistical index according to the time sequence of their occurrence [12]. Its formation is very simple, but it is widely used, and the process and phenomenon of the development of many things can be expressed by it, like the annual precipitation in meteorological statistics, in industrial production, the number of finished products in each quarter, in the field of national economy, annual GDP, and so on; the research fields involved in time series are numerous.

In people's research on time series, there are generally two purposes: one is to establish a model to understand the mechanism of generating the time series; and the second is to predict the possible level of the time series in the next time period based on the historical data of the series and some other influencing factors.

\section{(2) Basic Concepts of Time Series.}

Step 1: Stationary process: It is a special stochastic process whose main characteristic is that its statistical characteristics are fixed with time.

Let $\left\{X_{t}\right\}$ be a time sequence; for all positive integers $r$, take $t_{1}, t_{2}, \ldots, t_{r} \in T$ for any integer $t$; there are

$$
F_{t_{1}, t_{2}, t_{3}, \ldots, t_{r}}\left(x_{1}, x_{2}, x_{3}, \ldots, x_{r}\right)=F_{t_{1}+\tau, t_{2}+\tau, t_{3}+\tau, \ldots, t_{r}+\tau}\left(x_{1}, x_{2}, x_{3}, \ldots, x_{r}\right) \text {. }
$$

Then $\left\{X_{t}\right\}$ is called a strictly stationary time series.

In our practical application, it is very difficult to obtain the distribution of random sequences; even if we know the joint distribution, it is very difficult to apply on its basis. Therefore, the discussion of Yan Ping is generally studied at the theoretical level. In practical applications, more people will choose wide stationary sequences with relatively loose restrictions.

Let $\left\{X_{t}\right\}$ be a time series if the following conditions are met:

(1) Take $t \in T, E X_{t}^{2}<\infty$.

(2) Take $t \in T$; there is $E X_{t}=\mu=\mu$, where $\mu$ is a constant.
(3) The time series $\left\{X_{t}\right\}$ is called a broadly stationary time series if $r, s, t \in T$ has $\gamma(r, s)=\gamma(t, t+s-r)$.

Generally speaking, a time series that satisfies the strict stationary condition must satisfy the wide stationary condition, and vice versa. But this is not absolutely true. There are special examples in these two aspects. When the time series satisfies the condition of normal distribution, they are consistent.

Pure random sequence (also called white noise sequence) is a special stationary time series. White noise test is very important in time series, and it is an important basis to judge the rationality of the model. The definition of pure random sequence is introduced below.

Sequence $\left\{X_{t}\right\}$ satisfies the following conditions:

(1) Take $t \in T, E X_{t}=\mu$.

(2) If $t, s \in T$ has $\left\{\begin{array}{ll}\sigma^{2}, & t=s, \\ 0, & t \neq s\end{array}\right.$, then $\left\{X_{t}\right\}$ is said to be a pure random sequence.

(3) Characteristic statistics.

Some commonly used low-order and correlation functions are as follows.

Taking any time series $\left\{X_{t}, t \in T\right\}$, the mean value function is defined as

$$
\mu_{t}=E\left(X_{t}\right)=\int_{-\infty}^{\infty} x \mathrm{~d} F_{t}(x)
$$

where $F_{t}(x)$ is the distribution function of $X_{t}$ and $\mu_{t}$ is the mean value function of sequence $\left\{X_{t}\right\}$ at $t$.

The variance function is defined as follows when there is $\int_{-\infty}^{\infty} x \mathrm{~d} F_{t}(x)<\infty$ :

$$
D X_{t}=E\left(X_{t}-\mu_{t}\right)^{2}=\int_{-\infty}^{\infty}\left(X_{t}-\mu_{t}\right) 2 \mathrm{~d} F_{t}(x)
$$

Autocovariance is defined as

$$
r(t, s)=E\left(X_{t}-\mu_{t}\right)\left(X_{s}-\mu_{s}\right) .
$$

The autocorrelation coefficient is defined as

$$
\rho(t, s)=\frac{r(t, s)}{\sqrt{D X_{t} \cdot D X_{s}}}
$$

The closer the autocorrelation coefficient is to zero, the weaker the sequence correlation is, and the closer it is to $l$, the stronger the sequence correlation is.

\subsubsection{Stationary Time Series Model}

(1) AR Model. If the time series model satisfies the following structural conditions, it is called $p$-order autoregressive model, abbreviated as $\operatorname{AR}(P)$ :

$$
\left\{\begin{array}{l}
x_{t}=\varphi_{0}+\varphi_{1} x_{t-1}+\varphi_{2} x_{t-2}+\cdots+\varphi_{p} x_{t-p}+\varepsilon_{t}, \\
E\left(\varepsilon_{t}\right)=0, \operatorname{Var}\left(\varepsilon_{t}\right)=\sigma_{\varepsilon}^{2}, E\left(\varepsilon_{t} \varepsilon_{s}\right)=0, \\
E\left(\varepsilon_{s} \varepsilon_{t}\right)=0 .
\end{array}\right.
$$


(2) MA Model Q. -order moving average model, abbreviated as MA (Q), has the following structure:

$$
\left\{\begin{array}{l}
x_{t}=\mu+\varepsilon_{t}-\Theta_{1} \varepsilon_{t-1}-\Theta_{2} \varepsilon_{t-2}-\cdots-\Theta_{q} \varepsilon_{t-q}, \\
E\left(\varepsilon_{t}\right)=0, \operatorname{Var}\left(\varepsilon_{t}\right)=\sigma_{\varepsilon}^{2}, E\left(\varepsilon_{s} \varepsilon_{t}\right)=0 .
\end{array}\right.
$$

(3) ARMA Model. Autoregressive moving average model, abbreviated as $\operatorname{ARMA}(P, Q)$, has the following structure:

$$
\left\{\begin{array}{l}
x_{t}=\varphi_{0}+\varphi_{1} x_{t-1}+\varphi_{2} x_{t-2}+\ldots+\varphi_{p} x_{t-p}+\varepsilon_{t}-\Theta_{1} \varepsilon_{t-1}-\Theta_{2} \varepsilon_{t-2}-\cdots-\Theta_{q} \varepsilon_{t-q}, \\
\left(\varepsilon_{t}\right)=0, \operatorname{Var}\left(\varepsilon_{t}\right)=\sigma_{\varepsilon}^{2}, E\left(\varepsilon_{s} \varepsilon_{t}\right)=0, \\
E\left(\varepsilon_{s} \varepsilon_{t}\right)=0 .
\end{array}\right.
$$

2.3.3. Nonstationary Time Series Model. Because the sequences obtained in practical applications are usually unstable sequences and the stability of time series is required in the traditional linear model introduced earlier, the unstable sequences cannot be directly applied to the time series analysis model introduced above. This requires a method of converting an unstable sequence used to apply the ARMA model to the obtained stable sequence into a stable sequence, wherein the process of first transforming the sequence to fit the ARMA model is the ARIMA model as applied herein.

(1) ARIMA Model. Differential autoregressive integrated moving average model, abbreviated as $\operatorname{ARIMA}(P, D, Q)$ model, has the following structure:

$$
\left\{\begin{array}{l}
\varphi(B) \nabla^{d} X_{t}=\theta(B) \varepsilon_{t} \\
\left(\varepsilon_{t}\right)=0, \operatorname{Var}\left(\varepsilon_{t}\right)=\sigma_{\varepsilon}^{2}, E\left(\varepsilon_{s} \varepsilon_{t}\right)=0 \\
E\left(\varepsilon_{s} \varepsilon_{t}\right)=0
\end{array}\right.
$$

When $d=0$, model $\operatorname{ARIMA}(p, d, q)$ is actually model $\operatorname{ARIMA}(p, q)$. Fitting $\operatorname{ARIMA}(P, Q)$ model with $D$-order difference for nonstationary sequence is equivalent to fitting $\operatorname{ARIMA}(P, D, Q)$ model for original nonstationary sequence. The following summarizes the transformation process between the above time series models, as shown in Figure 3.

According to Cramer decomposition theorem, for any time series, its fluctuation is not only the result of random influence but also the result of deterministic influence.

It is worth noting that although the difference operation can fully extract the deterministic trend of time series, the nonstationary sequence is transformed into a stationary sequence, but every difference will be accompanied by certain information loss. Therefore, when applying this method of difference operation, it usually follows the principle of "being low but not high"; if you can use loworder difference, you will not use high-order difference.

\subsubsection{ARIMA Modeling Steps}

Step 1. The common inspection methods for stationarity inspection are as follows:
(1) Sequence diagram test method:Time sequence diagram is an indispensable part of time series analysis. By making a time series diagram to observe the data distribution, if the data distribution fluctuates around a constant value, it can be roughly judged that the time series is stationary. Although this method is simple and convenient, it has great subjectivity and is not accurate enough. Therefore, it can only be used as a preliminary judgment.

(2) Unit root test:PP test is mainly suitable for the case of nonhomogeneous variance; DF test is used in the case of first-order autoregression; $\mathrm{ADF}$ test is mainly applicable to the case of homogeneous variance.

(3) Correlation function judgment method:The sample autocorrelation function is

$$
\widehat{\rho}_{k}=\frac{\widehat{\gamma}_{k}}{\widehat{\gamma}_{0}}=\frac{\operatorname{cov}\left(X_{t}, X_{t-k}\right)}{\sigma_{X_{t}}, \sigma_{X_{t-k}}}=\frac{\sum_{i=1}^{n=k}\left(X_{t}-\bar{X}\right)\left(X_{t-k}-\bar{X}\right)}{\sum_{i=1}^{n=k}\left(X_{t}-\bar{X}\right)^{2}} \text {. }
$$

Observe the ACF diagram. When $k$ increases, if $\widehat{\gamma}_{k}$ is in a state of rapid attenuation, it is judged to be stable. If $\widehat{\gamma}_{k}$ shows a slow decay state, it is judged to be nonstationary.

Step 2. Smoothing:If the test result in Step 1 is nonstationary, it needs stationary treatment. Generally, there are two ways: one is direct difference treatment, and the other is logarithmic treatment before difference treatment. If the original sequence is stationary, Step 3 is directly carried out.

Step 3. Pure random test:The data processed in Step 2 is stable, and then white noise test is done. If the sequence is white noise sequence, the modeling is finished; otherwise, go to the next step.

Step 4. Identifying the model structure:Observe the sample ACF graph and PACF graph to identify the model and determine the order, and determine the range of model parameters. The basic principles of model recognition and ranking are shown in Table 1 . It should be noted that this model identification and 


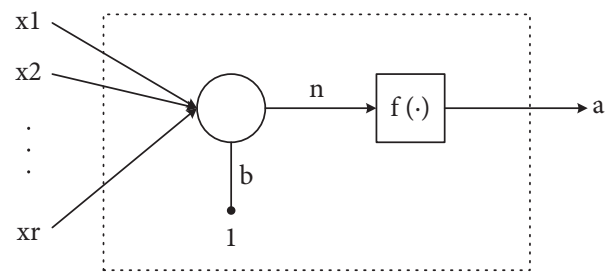

Figure 1: Neuron structure.

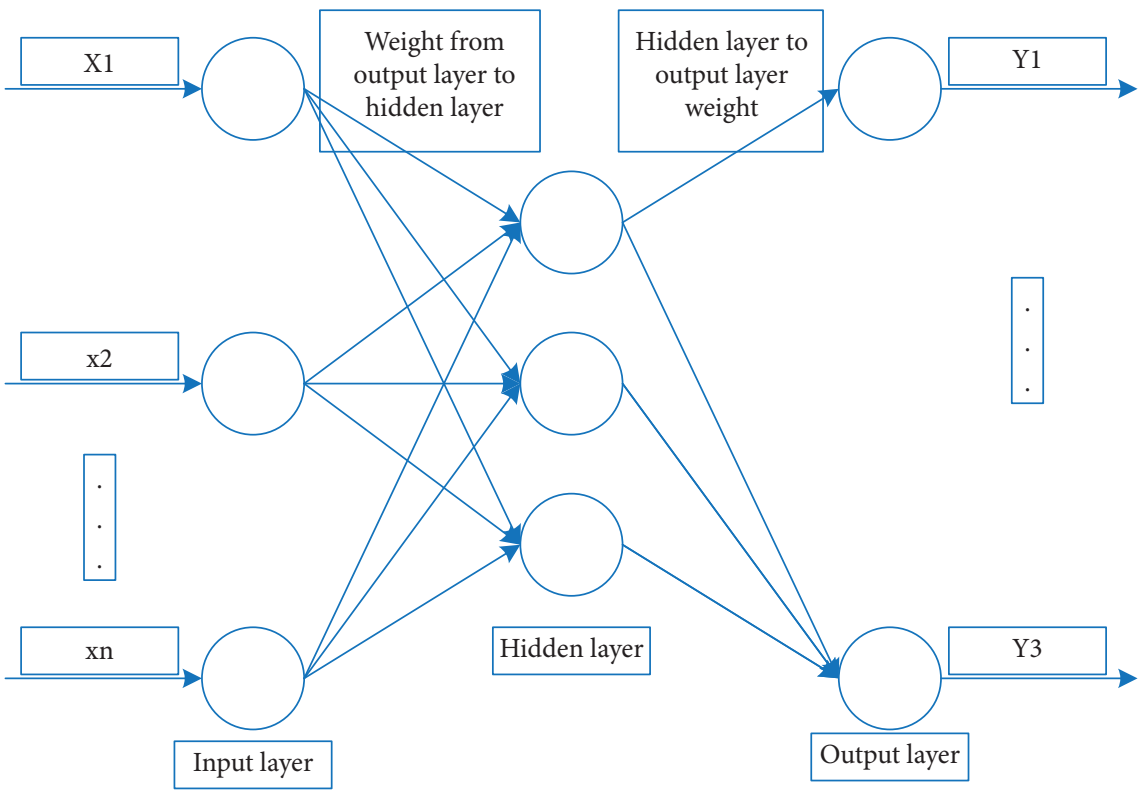

Figure 2: Topological structure of BP neural network.

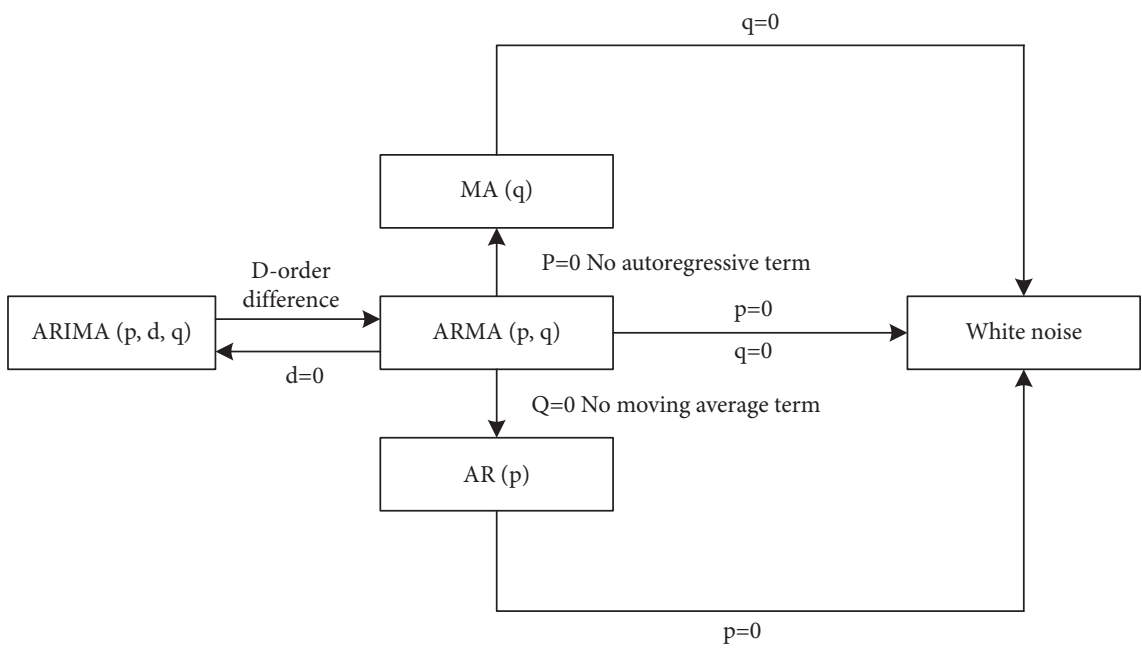

Figure 3: Model transformation diagram.

TABLE 1: Model identification criteria.

\begin{tabular}{lcc}
\hline ACF & PACF & Selection model \\
\hline Tailing & $P$-order truncation & AR $(P)$ \\
$Q$-order truncation & Tailing & MA $(q)$ \\
Tailing & Tailing & ARMA $(p, q)$ \\
\hline
\end{tabular}


order determination method is subjective and not very accurate, so we calculate the AIC value and BIC value of the model corresponding to each group of parameters within the range of model parameters and find the optimal model according to AIC criterion and BIC criterion.

AIC criterion (Minimum Information Criterion), which is based on the prediction error of the model and is used to judge whether the lag order finally determined by the autoregressive model is appropriate, can be expressed as

$$
\mathrm{AIC}=-2 \ln (L)+2 k
$$

where $L$ is the exponential likelihood function and $K$ is the number of selected parameters.

Step 5. Model validation:It is divided into parameter significance and model adaptability test. The former is to test whether the parameter is significantly zero to judge whether the independent variable corresponding to a parameter has a significant effect on the model and then consider whether to eliminate the variable. The latter is to test whether the residual sequence is white noise sequence and then judge whether the model is valid.

Step 6. Prediction and analysis of the model

The model is used to predict the short-term sequence values in the future. The modeling process of ARIMA model is shown in Figure 4.

\subsection{Hybrid Model of Neural Network and ARIMA}

2.4.1. Advantages of Hybrid Models. A hybrid model is a new model formed by combining different models. From the perspectives of model itself and data itself, on one hand, there are many models in the field of forecasting; different models have their own advantages and disadvantages. At the same time, there are many similarities between different models, which are not opposite. In order to be compatible with the advantages of different models, different models can be combined to construct a new model. Because the new model has the advantages of two or more models at the same time, the final prediction effect is more ideal. On the other hand, in practice, most time series contain both linear and nonlinear components. In this case, the mining of information by a single model is limited, which often cannot achieve the best prediction effect. Then, combining the model suitable for linear fitting with the model suitable for nonlinear fitting makes the hybrid model have both linear and nonlinear processing abilities, thus making the prediction effect more ideal. We know that neural network is very good at data processing, and learning ability is also the advantage of neural network, which is good at mining nonlinear features of sequences. Neural network also has certain advantages in operation. First, it requires simple number of experimental samples; second, its structure is flexible and its design is convenient. However, ARIMA model, a traditional time series model, is good at mining linear features of data. Then, this section aims to combine a variety of neural network models with the ARIMA models to form a hybrid model of multiple neural network models and ARIMA models, so that the hybrid model has both linear processing ability and nonlinear processing ability. Finally, the constructed hybrid model is applied to the prediction of enterprise economic data.

2.4.2. Introduction to Mixed Modeling Methods. The hybrid model can be divided into two hybrid methods: The first method is to combine different models through the method of weight allocation. Because the prediction results of different models are different, it is necessary to allocate the weights of the models participating in the combination reasonably, so the new mixed model is formed by the method of allocating different weights to different models. For this combination, the distribution of weights should be set in advance and should be reasonable and effective. The second method is to use model $x_{1}$ to predict and get prediction result 1 and residual error and then use the residual error as the input of model $x_{2}$ to predict and get result 2 and finally combine result 1 and result 2 . Superposition is used as the prediction result of the hybrid model. Compared with the first method, the advantage of this method is that it does not need to set reasonable and effective weight distribution in advance, and the disadvantage is that the data processing will be relatively complicated. This paper adopts the second combination mode. According to the ability of neural network model and ARIMA model, they are combined to form a hybrid model of neural network and ARIMA. Then, among the different data predictions applied by the hybrid model, the combination method is as follows.

Firstly, the ARIMA model is used to model the data, and the linear characteristics of the data are mined. The predicted value is recorded as $L(1)$, and the residual $e_{t}$ is calculated from the original sequence $y_{t}$ and $L^{\prime}(t)$; namely,

$$
e_{t}=y_{t}-L^{\prime}(t)
$$

Sequence $e_{t}$ contains the nonlinear characteristics of the original sequence $y_{t}$, so the nonlinear characteristics of the data are further mined, and the neural network model is applied to approximate this nonlinear relationship in $e_{t}$; that is, sequence $e_{t}$ is used as the input of the neural network after matrix transformation and normalization, and the output result $M^{\prime}(t)$ is obtained. Finally, the two are added as the prediction result $N^{*}(t)$ of the mixed model:

$$
N^{\prime}(t)=L^{\prime}(t)+M^{\prime}(t) .
$$

At this time, the prediction effect of the hybrid model is compared with that of the single neural network model in the previous paper.

\subsubsection{Concrete Steps of Hybrid Prediction Model Modeling.}

Step 1: Establish ARIMA time series model for original data. The ARIMA model is used to fit the rule of sample 


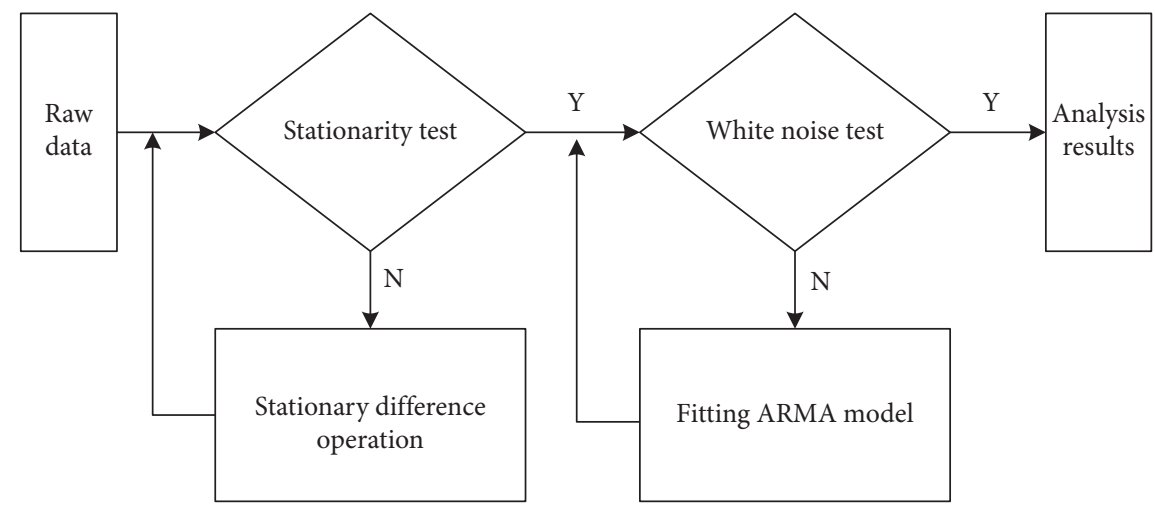

FIGURE 4: ARIMA model modeling process.

data, and the future data is predicted to get the predicted value and residual error.

Step 2: Establish three neural network models for the residuals obtained in Step l. Firstly, the training set and test set are generated by the sliding window method and then normalized as the input matrix of single neural network prediction model, and then the network is trained to predict and get the predicted value.

Step 3: Add the prediction results in the previous two steps, respectively, to obtain the final prediction values of the three mixed models. The hybrid model flow is shown in Figure 5.

\section{Case Analysis of Economic Audit Forecast of Sales Business of Commodity Circulation Enterprises}

The main business process of commodity circulation enterprises is procurement-warehouse-sales-transportation, and whether the sales data is accurate and effective directly affects the authenticity of enterprise interests. In addition, the correctness of sales forecast is very important for enterprises to make purchase decisions and sales plans. Inappropriate purchases lead to inventory backlog and other problems, which affect the capital flow of enterprises. Therefore, it is meaningful to audit sales data.

This paper validates the application effect of outlier analysis method and the mixed model of neural network and ARIMA in the business audit of commodity circulation enterprises by using the business case data of Y Enterprise.

3.1. Business Background. Y Enterprise was established in 1992, headquartered in Chongqing. It is mainly engaged in the procurement, sales, and distribution of electronic products, parts, and supporting products. As a chain enterprise, Y Enterprise has the operating characteristics of "integrating business flow, logistics and information flow" and will integrate the vertical chain management of "taking the head office as the axis, going forward to various suppliers, middle and chain stores, and going back to consumers." In terms of operation, it adopts the mode of "unified procurement of the company-unified distribution of distribution centers-sales of several chain stores." Finance adopts the method of "unified price setting, unified calculation, and unified tax payment." In terms of service, the standard of "unified service for all stores" is established and the "six unifications" of operation mode are realized as a whole. Y Enterprise's asset management focuses on various electronic products, and the capital flow is relatively simple. Buy goods with money, and then turn them into money after being sold, that is, "currency-commodity-currency." Enterprises obtain the intermediate difference in the mode of "buy low and sell high" and deduct various expenses to make profits. That is to say, buying goods from manufacturers, providing distribution to consumers, setting up services, selling various goods, and earning differences have the characteristics of commodity circulation enterprises.

Y Enterprise has developed and built a management information system with enterprise characteristics and realized management informationization and financial informationization, but the audit work still stays in the traditional manual audit stage. One is that enterprises do not have special monitoring software, so they assist in monitoring business. Inspectors can use the information management system to investigate the shared accounting and banking flows on the platform, but the management system can initially find out the suspected evidence. Second, inspectors have no information foundation, and the level of computers is limited. According to business experience, data are compared, analyzed, checked, and confirmed.

3.2. Audit Planning Phase. Due to the limitation of data acquisition, the audit objectives of this case mainly have two points: (1) Detect abnormal audit data, delete abnormal data and select them, and find out the doubtful points of audit. (2) Make sales forecast to judge whether the predicted value in the enterprise sales plan is reliable, and take it as one of the references for whether the enterprise sales plan is effective.

The analysis of this example is based on audit objectives; the original sales data is collected from the marketing management information system of Y Enterprise by SQL sentence. According to the outlier algorithm and the data analysis requirements of neural network and ARIMA mixed model, the original sales data is pretreated by Python tool, and the pretreated data is applied to the two algorithm 


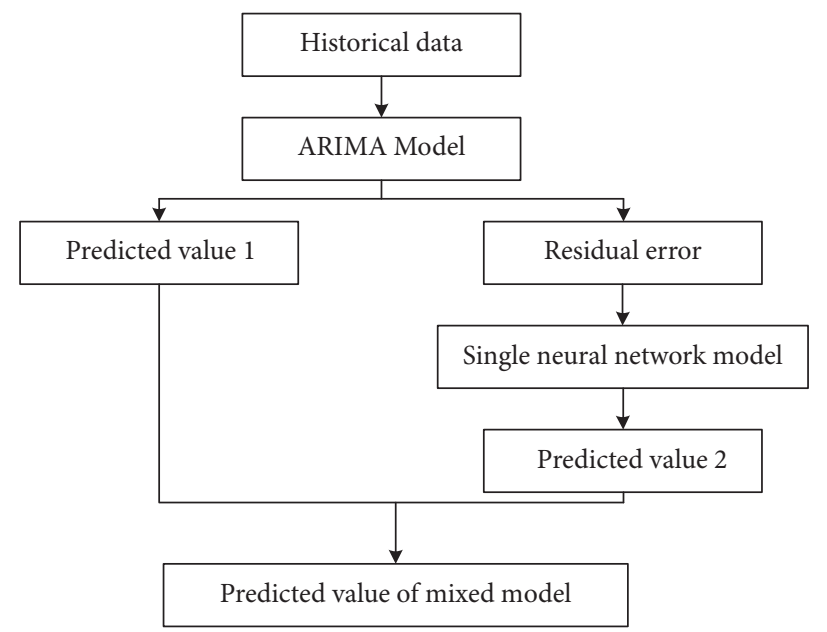

FIGURE 5: Flow chart of mixed prediction model.

models for data analysis. The specific audit procedure is shown in Figure 6.

\subsection{Audit Data Analysis based on Neural Network and} ARIMA Hybrid Model. For Y Enterprise, the reliability of sales plan directly affects the economic effect of the enterprise the next year. Therefore, doing a good job in reviewing the sales plan is conducive to improving the economic benefits of enterprises. This time, using the actual sales volume of Y Enterprise from January 2019 to June 2020, a hybrid model of neural network and ARIMA is constructed to predict the sales volume of the model from July to December 2020. The predicted results are compared with the actual sales volume and the predicted values of enterprise sales plan to verify the feasibility and effectiveness of the hybrid model of neural network and ARIMA. It provides a new method for the inspector to review the sales plan.

\subsubsection{Application of Hybrid Model of Neural Network and} ARIMA. (1) Stationarity Test. In this section, the stability of predicted samples is checked according to ADF test method by using time series diagram. In the historical sales data sequence diagram in Figure 7, the data is checked unilaterally using the $\mathrm{ADF}$ check method, and its $P$ is judged. If the value is less than 0.05 , the sequence is stable. The value of the received sample is 0.994 , which is greater than 0.05 , so the original sequence is unstable.

(2) Differential Conversion. The unsteady sample sequence is derived from the time series diagram of the data after differential transformation by the first-order difference in Figure 8, and the ADF check is carried out on the sequence, and the value obtained by $p$ is $5.057 e-11$, which is less than 0.05 . Therefore, the sequence after primary difference is a stable sequence, which is called primary difference sequence, and the next parameter estimation can be carried out.

(3) Parameter Estimation. Autocorrelation and deflection autocorrelation analyses are carried out for the first difference sequence. According to the correlation diagram and deflection autocorrelation diagram, as shown in Figures 9 and 10, it can be seen that the autocorrelation value is selected to be of order 2 and the partial autocorrelation value is selected to be of order 3 .

Using BIC matrix to test parameters, the optimum values of $P$ and $Q$ are 3 and 3, respectively, so the optimum mixed model of neural network and ARIMA is ARIMA (3, $1,3)$.

(1) Model checking:The partial correlation coefficient is used to test the residual correlation, and the test result is 1.77 , which shows that there is no autocorrelation and it can be effectively predicted.

(2) Model prediction:Use ARIMA $(3,1,3)$ to forecast the sales volume from July to December 2020, and the results are shown in Figure 11.

\subsection{Analysis of Application Results of Hybrid Model of Neural} Network and ARIMA. Using neural network and ARIMA hybrid model to forecast and analyze the sales business data, the predicted value of the model, the actual value, and the predicted number in the sales plan are compared and analyzed to judge the effectiveness of the application of the model. A specific comparison is shown in Figure 12.

Generally speaking, the prediction results of neural network and ARIMA hybrid model are close to the actual value, and the prediction results are more reliable than those in the sales plan. According to the survey, Y Enterprise has complete purchasing and preentry management information system. When carrying out sales forecast, according to the experience of the salesperson in charge, it makes a simple linear analysis of sales data to get the forecast result, which is the basis of sales plan. The prediction results of the hybrid model of neural network and ARIMA are more accurate and reliable than the existing sales prediction methods of enterprises, so the hybrid model of neural network and ARIMA can be used to review the sales prediction of enterprises.

The reliability of sales forecast has an important influence on the purchasing arrangement, inventory 


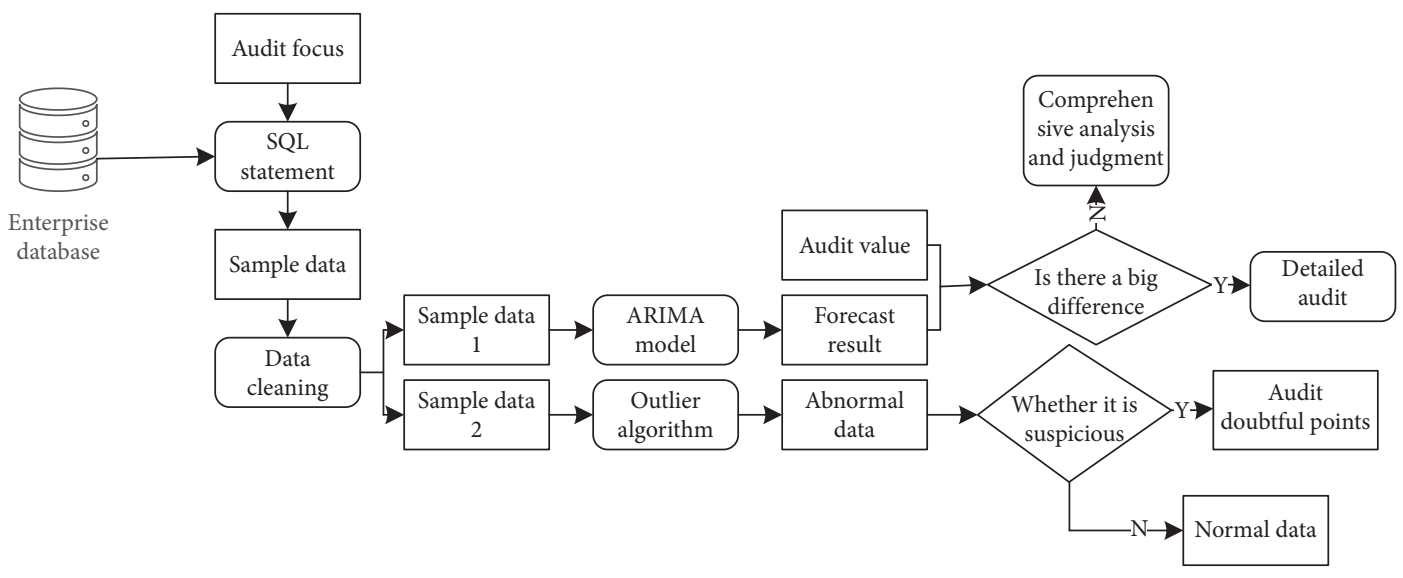

Figure 6: Audit program design.

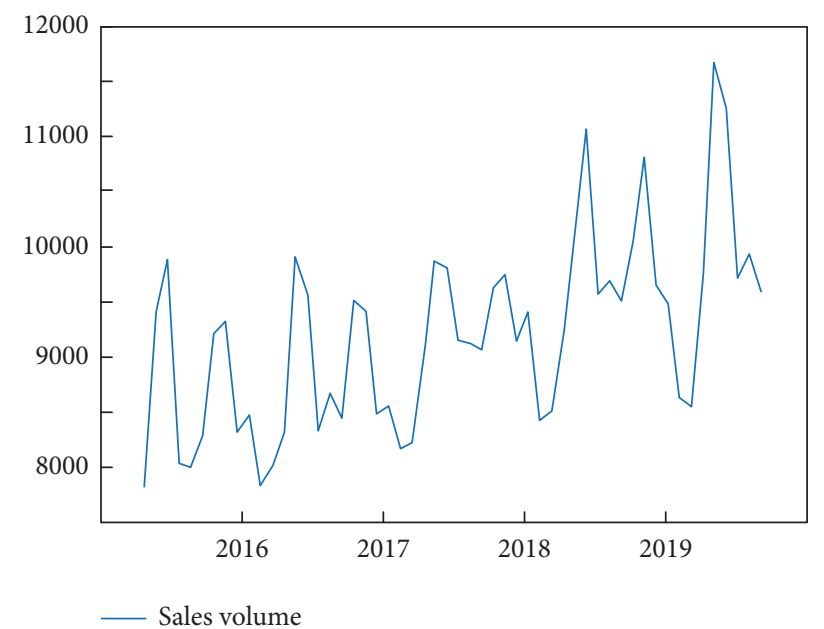

FIGURE 7: Sequence diagram of sample sequence.

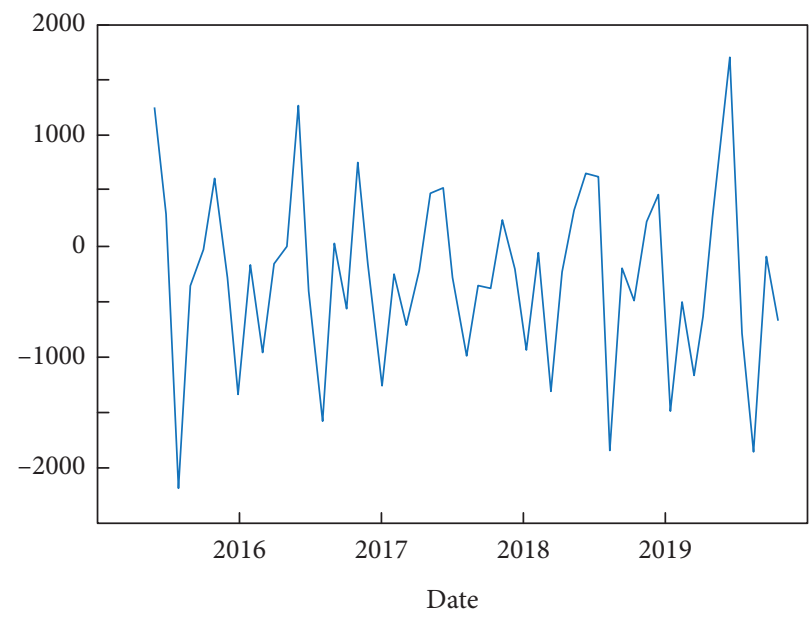

- First-order difference

Figure 8: First-order difference sequence diagram. 


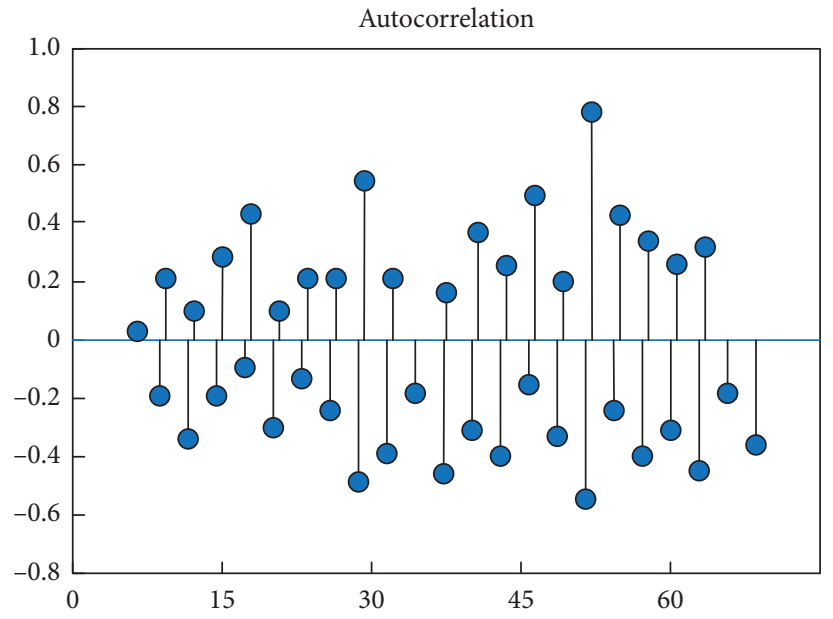

FIgURE 9: Autocorrelation diagram of first-order difference sequence.

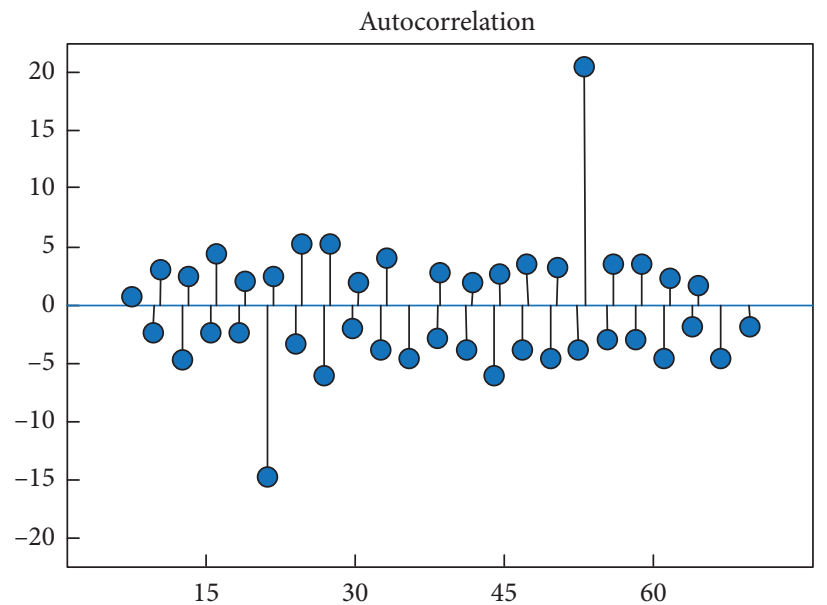

Figure 10: Partial autocorrelation diagram of first-order difference sequence.

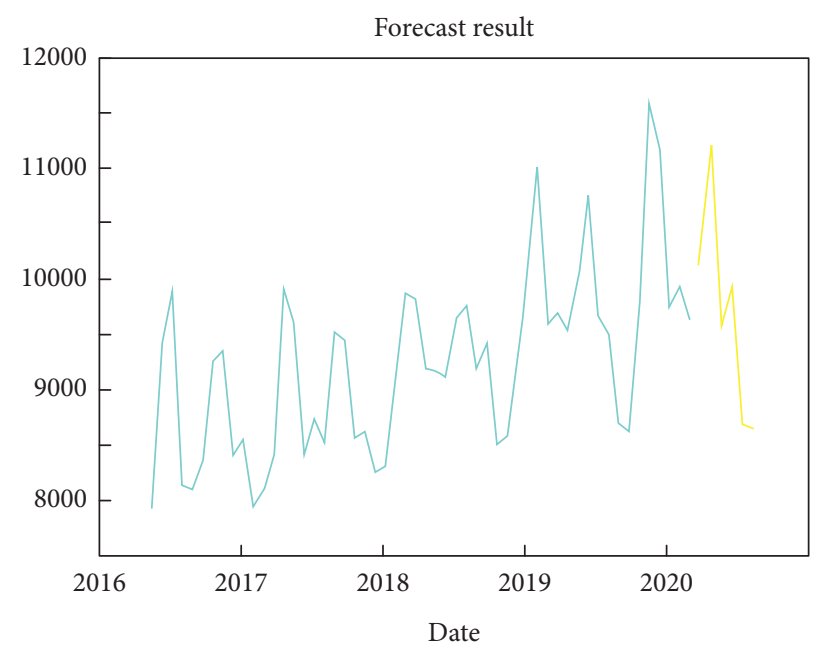

Figure 11: Sales forecast results from July to December 2020. 


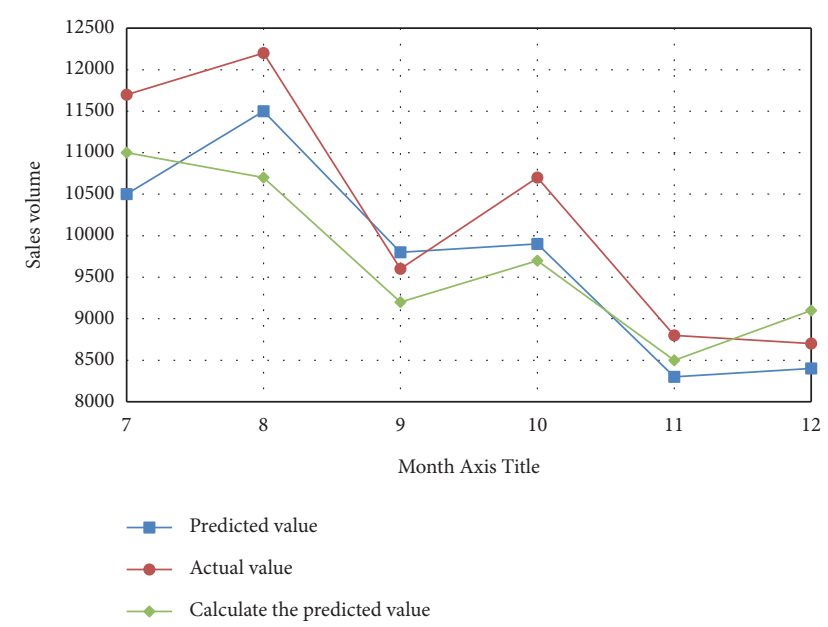

FIGURE 12: Comparison of predicted value, actual value, and planned predicted value.

management, and distribution of sales resources. The examination of sales forecast has an effect on improving the business performance of enterprises and can effectively prevent the possible business risks of enterprises in the future.

When the inspector reviews the forecast value of the sales plan by using the hybrid model of neural network and ARIMA, if the difference between the forecast result of the model and the forecast value of the plan is large, it is necessary to review the sales plan in detail during the forecast period. This is mainly reflected in the following aspects: (1) whether the market demand is considered; (2) whether the market competition situation is analyzed in detail, especially the sales situation of competitors; (3) whether the completion of the previous sales plan has been summarized, and, on the basis of the summary, the existing sales forecasting methods have been optimized; and (4) whether the changes in the sales force are considered. Therefore, auditors can comprehensively analyze the effectiveness of the enterprise sales plan in combination with the above situation and judge whether there are factors such as large changes in organizational personnel and changes in market demand, which lead to the discrepancy between the planned forecast value and the previous sales data rules.

\section{Data Availability}

The experimental data used to support the findings of this study are available from the corresponding author upon request.

\section{Conflicts of Interest}

The authors declare that they have no conflicts of interest regarding this work.

\section{References}

[1] P. Shahnoori and G. P. Jenkins, "Valuation of the quality attributes of online banking services by small and medium enterprises engaged in international trade," South African Journal of Economics, vol. 87, no. 1, pp. 65-81, 2019.

[2] M. D. Liu, L. Ding, and Y. L. Bai, "Application of hybrid model based on empirical mode decomposition, novel recurrent neural networks and the ARIMA to wind speed prediction," Energy Conversion and Management, vol. 233, no. FEB, Article ID 113917, 2021.

[3] D. Li, L. Deng, and Z. Cai, "Statistical analysis of tourist flow in tourist spots based on big data platform and DA-HKRVM algorithms," Personal and Ubiquitous Computing, vol. 24, no. 1 , pp. $87-101,2020$.

[4] F. Willnecker and H. Krcmar, "Model-based prediction of automatic memory management and garbage collection behavior," Simulation Modelling Practice and Theory, vol. 93, pp. 164-191, 2019.

[5] Z. Xin, X. A. Sheng, A. L. M. Vilela, and H. Eugene Stanley, "Inter-event time interval analysis of organizational-level activity: venture capital market case," Physica A: Statistical Mechanics and Its Applications, vol. 516, pp. 346-355, 2019.

[6] J. Yuan, "Research on behavior prediction based on deep learning-take chengdu economic innovation enterprise as an example," E3S Web of Conferences, vol. 275, no. 1, Article ID 03060, 2021.

[7] Z. Sun, Z. Lei, J. Zou, R. Bai, H. Jiang, and C. Yan, "Prediction of failure behavior of composite hat-stiffened panels under inplane shear using artificial neural network," Composite Structures, vol. 272, p. 15, Article ID 114238, 2021.

[8] F. Ciampi, V. Cillo, and F. Fiano, "Combining Kohonen maps and prior payment behavior for small enterprise default prediction," Small Business Economics, vol. 54, no. 4, pp. 1007-1039, 2020.

[9] S. Y. Liu, S. Liu, Y. Tian, L. Quan, and Y. Y. Tang, "Research on forecast of rail traffic flow based on ARIMA model," Journal of Physics: Conference Series, vol. 1792, no. 1, Article ID 012065, 2021.

[10] Y. Zheng, K. Yuan, and L. Dai, "Research on coal price forecast based on ARIMA and SVM combination model," E3S Web of Conferences, vol. 257, no. 26, Article ID 02008, 2021.

[11] J. Wang, J. Cheng, F. Liu, Y. Lei, and T. Taijie, "Research on the air quality prediction model of Wuhai mining area based on deep learning," E3S Web of Conferences, vol. 300, no. 2, Article ID 02005, 2021.

[12] X. Wang, B. Guo, X. Ma, and B. Xuemei, "Research on emotional music reconstruction method based on DBNGRU," Journal of Physics: Conference Series, vol. 1966, no. 1, Article ID 012016, 2021. 